\title{
ANÁLISE DA RENDA AGRÍCOLA DOS AGRICULTORES EM UM MUNICÍPIO NA REGIÃO DO MÉDIO PARANAPANEMA
}

\author{
Vinícius Rafael Bianchi* \\ Leonardo de Barros Pinto** \\ Sandra Cristina de Oliveira***
}

RESUMO: O principal objetivo deste trabalho foi analisar a renda agrícola dos agricultores do município de Palmital (SP), localizado na região do Médio Paranapanema. Por meio de análise estatística multivariada (modelo de regressão múltiplo) identificou-se os fatores que interferem positiva ou negativamente na renda agrícola dos agricultores. Os dados foram coletados por meio de um formulário de pesquisa aplicado a uma amostra de agricultores estratificada, proporcional à condição do estabelecimento rural (familiares e patronais), dados correspondentes ao período de agosto de 2013 a julho de 2015. Destaca-se que este trabalho possui caráter interdisciplinar, abordando aspectos qualitativos e quantitativos, cuja análise estatística trabalhou com quarenta e nove variáveis destacando-se os sistemas de produção encontrados. Tal análise permitiu identificar as variáveis que mais afetam positiva ou negativamente a renda agrícola destes agricultores. Como resultado, observou-se que há indícios de que a diversidade dos sistemas de produção melhora a renda dos agricultores familiares, podendo significar que o aumento de um desses sistemas, ou seja, a atribuição do mesmo a mais áreas, poderá contribuir positivamente à renda agrícola destes agricultores. Em relação aos agricultores patronais, identificou-se que variáveis como tamanho da área e produção por hectare foram fundamentais para a renda agrícola destes agricultores no município estudado.

PALAVRAS-CHAVE: Agricultura familiar; Agricultura patronal; Análise estatística; Interdisciplinaridade; Renda.

\footnotetext{
"Doutorando em Agronomia pela Faculdade de Ciências Agronômicas - FCA da Universidade Estadual Paulista UNESP, Brasil. E-mail: vrbianchifca@gmail.com

${ }^{* *}$ Doutor em Engenharia Agrícola pela Universidade Estadual de Campinas - UNICAMP, Brasil.

**** Doutor em Ciências da Computação e Matemática Computacional pela Universidade de São Paulo - USP, Brasil.
} 


\title{
FARMERS` EARNINGS IN A MUNICIPALITY OF THE MID-PARANAPANEMA REGION
}

\begin{abstract}
Farmers' earnings in the municipality of Palmital, in the middle Paranapanema region, are analyzed. Multivariate statistical analysis (multiple regression model) identified the factors that positively or negatively interfere in the farmers' earnings. Data were retrieved by a survey among a stratified selection of farmers ranked according to rural establishments (familial and ownership), between August 2013 and July 2015. Current interdisciplinary research comprised qualitative and quantitative aspects whose statistical analysis involved 49 variables on production systems. Analysis identified the variables that most affected the farmers' earnings. Results revealed that diversity of production systems improved the profits of familial farmers. It may actually increase one of the systems, or rather, their attribution to further areas that may improve their earnings. In the case of farmer-owners, variables, such as area size and production per hectare, were crucial for their agricultural earnings.
\end{abstract}

KEY WORDS: Familial agriculture; Farm owners' agriculture; Statistical analysis; Interdisciplinarity; Earnings.

\section{INTRODUÇÃO}

O Produto Interno Bruto (PIB) do agronegócio representa cerca de 21,5\% do PIB total da economia brasileira (CEPEA, 2017), dados que evidenciam a importância deste setor para a economia, gerando empregos e renda direta e indiretamente para diversos setores no país.

Entende-se por renda a remuneração dos recursos produtivos utilizados na produção de bens e serviços de uma economia, da remuneração da mão de obra por meio de salário, onde "[...] maior será a renda de uma sociedade quanto mais recursos produtivos ela absorver" (NOGAMI, 2012, p. 18). A renda obtida nos estabelecimentos rurais é composta tanto pelas atividades agrícolas, quanto pelas atividades não agrícolas, estas últimas cada vez mais presentes no cenário da agricultura, além de outras fontes advindas de programas governamentais ou da aquisição de benefícios como, por exemplo, aposentadoria (NUNES, 2009).

A partir das diferentes configurações possíveis relacionadas à diversidade 
produtiva, somada as características da família e aos tipos de agricultores existentes em determinada localidade com os distintos sistemas de produção por eles desenvolvidos, é possível determinar quais os fatores que mais afetam a composição da renda agrícola.

Para os agricultores familiares e patronais, surge um problema de ordem socioeconômica. Pois, além de transformar os cenários das regiões rurais e urbanas, acaba na maioria das vezes prejudicando os agricultores rurais familiares, que não tendo capacidade de se manterem, migram para as cidades. Além disso, uma característica muito presente principalmente na agricultura familiar é a pluriatividade, a interação entre atividades agrícolas e não agrícolas ligadas às estratégias de sobrevivência das famílias e depende do contexto em que estas estão inseridas (SCHNEIDER, 2009). Já os agricultores patronais acabam sendo responsabilizados pela transformação desses cenários devido às mudanças em seus sistemas de produção, na maioria das vezes com monoculturas em larga escala, para atender as demandas de mercado.

O objetivo principal deste trabalho é identificar quais são os fatores sociais, econômicos e ambientais, por meio de variáveis inerentes ao perfil dos estabelecimentos rurais que interferem na renda agrícola dos distintos sistemas de produção desses estabelecimentos caracterizados como familiares ou patronais na região do Médio Paranapanema, no Estado de São Paulo, especificamente no município de Palmital. Para tanto são obtidos modelos de regressão linear múltipla que indicam os fatores ou a combinação deles que interfere de maneira positiva ou negativa à composição da renda agrícola dos agricultores familiares e patronais do município em estudo.

Situado no Centro-Oeste paulista, Palmital pertence à região do Médio Paranapanema, composta por 18 municípios da comarca da região de Assis, que possui economia basicamente agrícola, com destaque para o cultivo da cana de açúcar (UDAETA et al., 2004). Destaca-se também o cultivo da mandioca voltada à indústria, devido às condições edafoclimáticas da região (FURLANETO; KANTHACK; BONISSONI, 2006). Além das condições associadas aos aspectos produtivos, Palmital (SP) se beneficia por meio de questões logísticas, pois, encontra-se às margens da Rodovia SP 374, que facilita o acesso à capital paulista, aos Estados do Paraná e do 
Mato Grosso do Sul. Ademais, a região foi considerada a precursora das práticas de plantio direto (ÁREA..., 2000).

\section{FUNDAMENTAÇÃO TEÓRICA}

\subsection{AGRICULTURA FAMILIAR}

A origem da agricultura familiar no Brasil se deu por meio de cinco grupos, os índios, os escravos africanos, os mestiços, os brancos não herdeiros e os imigrantes europeus, segundo pesquisadores brasileiros (ALTAFIN, 2007).

Sua definição é bastante complexa, pois, envolvem vários fatores, como tamanho do estabelecimento rural, gestão do estabelecimento, mão de obra, origem da renda entre outros. As inúmeras possibilidades de composição da agricultura familiar, bem como as próprias diferenças existentes nos territórios, podem levar a formas de reprodução das unidades familiares exitosas ou não e, no caso negativo, com o agravante do risco de extinção destas ao longo do tempo (ABRAMOVAY, 2010).

Para efeitos de construção de uma definição geral (conceitualmente universalizável), capaz de abstratamente referenciar a extensa diversidade de situações históricas e socioeconômicas e de tipos econômicos, a agricultura familiar corresponde a formas de organização da produção em que a família é ao mesmo tempo proprietária dos meios de produção e executora das atividades produtivas. Esta condição imprime especificidades à forma de gestão do estabelecimento: referencia racionalidades sociais compatíveis com o atendimento de múltiplos objetivos socioeconômicos; interfere na criação de padrões de sociabilidade entre famílias de produtores; e constrange os modos de inserção, tanto no mercado produtor como no consumidor (NEVES, 2014, p. 47).

Envolvida pela diversidade, a agricultura familiar também se retrata pela desigualdade, nos mais diversos fatores como distribuição de terras, fatores tecnológicos, de implementos agrícolas, fatores de produção e produtividade, 
entre outros. É notável que a agricultura familiar seja excluída ao acesso de determinados recursos e serviços, que lhes seriam favoráveis na composição da renda agrícola. Além de que reduzir essa classe de trabalhadores a simples agentes econômicos é injusto mediante as desigualdades sociais que se tem no país. De forma sintética, é possível caracterizar e qualificar os agricultores familiares como:

[...] um grupo majoritário de estabelecimentos especializados, que depende muito fortemente da receita da atividade agropecuária [...] não são estabelecimentos cujas estratégias de reprodução vão passar pela produção, pois acessam muito pouco os serviços de extensão e possuem áreas de terras relativamente pequenas para ampliar a agricultura [...] que vivem no espaço rural, mas a agricultura e a produção agropecuária já não tem um sentido econômico e produtivo expressivo, mesmo assim, a produção continua a ter alguma importância, especialmente para o autoconsumo (DELGADO; BERGAMASCO, 2017, p. 96).

Neste contexto, a renda agrícola tem importância para sua manutenção e sobrevivência, questões econômicas estão em segundo plano mediante suas questões sociais.

\subsection{AGRICULTURA PATRONAL}

Apontado pelos censos agrícolas como a minoria em quantidade e detentores da maioria das terras, o agricultor patronal é caracterizado por produção de monoculturas em grande escala, produção mecanizada, maior tecnologia e voltado para o mercado. Deste modo, a agricultura se transformou em um grande agente econômico, com sistemas de produção que promovem desde a produção de insumos até a distribuição da produção agrícola (ZYLBERSZTAJN; NEVES; NEVES, 2005).

Agricultura patronal que também é chamada de agronegócio, em referência ao agribusiness, trata-se de um conceito que John Davis e Ray Goldberg utilizaram pela primeira vez nos Estados Unidos nos anos de 1950, relacionando economicamente o setor agropecuário com os demais setores, indústria, comércio e 
serviços (LEITE; MEDEIROS, 2012). Possui características peculiares em relação aos demais setores, que envolve sazonalidade, perecibilidade e heterogeneidade, além disso também se caracteriza pela integração de cadeias produtivas, de montante a jusante, com grandes multinacionais do setor investindo em inovação e garantindo pacotes tecnológicos que englobam desde maquinários agrícolas até pesquisas biotecnológicas (BUAINAIN et al., 2014).

A produção do agricultor patronal se faz em grandes escalas principalmente mundial em uma posição de poder, onde Nelson Delgado diz que:

Se fortalece desde o ajuste externo dos anos 1980 . embora tenha sido estimulado pela política estatal desde a modernização da agricultura na década de 1970 - e que ganha impulso, inclusive pela mídia, a partir do segundo governo Fernando Henrique Cardoso (DELGADO, 2012, p. 86).

\subsection{RENDA AGRÍCOLA}

A remuneração dos recursos produtivos na produção de bens e serviços de uma economia, ou seja, a remuneração da mão de obra por meio de salário é um dos componentes da renda que se forma juntamente com lucros, juros e alugueis, por exemplo (NOGAMI, 2012).

É complexo analisar a renda no meio rural, porém, é uma tarefa importante, pois, além de resultados econômicos, auxilia na compreensão de como os agricultores, principalmente os caracterizados como familiares, realizam suas escolhas e elaboram estratégias a partir de recursos disponíveis (NUNES, 2009).

A renda de estabelecimentos rurais é determinada não somente pelas características desses estabelecimentos e pelas atividades agrícolas neles desenvolvidas, ou seja, aquelas atividades relativas ao sistema de produção que geram renda agrícola, mas também pelas atividades que não têm relação com o sistema de produção e que geram renda não agrícola (PINTO et al., 2016).

Neste contexto observa-se que a renda rural ultrapassa a remuneração pelas atividades agrícolas, sua composição "[...] é dada pelas somas das rendas agrícolas e não agrícolas, além de outras fontes advindas de programas de governo ou de 
benefícios adquiridos como as aposentadorias" (NUNES, 2009, p. 258).

Já a renda agrícola é obtida pelas atividades agropecuárias de um estabelecimento rural. Lima et al. (2005, p. 75) dizem "[...] que a renda agrícola representa o valor agregado que fica com o agricultor para remunerar o trabalho familiar e aumentar seu patrimônio".

Em virtude da evolução econômica e tecnológica, a característica dos estabelecimentos rurais e a composição campo/cidade mudaram nos últimos 50 anos, a população urbana brasileira avançou de 30\% para 80\% (IBGE, 2012). Portanto, a mudança no cenário da agricultura suscita a hipótese de que há inúmeras combinações passíveis de análise sobre o sucesso de um estabelecimento rural, respeitando acima de tudo as novas formas de gestão, que na geração de renda estejam presentes nas atividades agrícolas e não agrícolas (PINTO et al., 2016).

Desta forma, a renda de estabelecimentos rurais tem sido objeto de vários estudos no Brasil (SILVA et al., 2008; MOCHIUTI et al., 2011; PINTO et al., 2016; dentre outros), visando conhecer a sua formação e buscar alternativas de geração de emprego e renda para o meio rural.

Silva et al. (2008) analisaram a rentabilidade de pequenas propriedades rurais pertencentes ao município de Painel (SC), utilizando-se de análise de regressão múltipla, com o objetivo de avaliar os fatores que são determinantes na formação da renda bruta das propriedades rurais estudadas.

Já Mochiuti et al. (2011) desenvolveram um estudo, que por meio da ferramenta estatística de análise de regressão múltipla, analisou a composição da renda nos estabelecimentos rurais do município de Tupã (SP), que apontou os fatores que interferiam positiva e negativamente à composição da renda bruta total (RBT), destacando a utilização das áreas para reforma de pastagens.

Enquanto Pinto et al. (2016) utilizaram da ferramenta estatística de análise de regressão múltipla, tendo como variável resposta a renda bruta total (RBT), para desenvolver um estudo em três municípios da Região Nova Alta Paulista. No caso deste trabalho, foram analisados somente os fatores que podem afetar a renda agrícola, e a remuneração trabalhada teve origem do capital da "terra". 


\section{MATERIAIS E MÉTODOS}

\subsection{COLETA DE DADOS}

A coleta de dados foi feita por um levantamento de informações concernentes aos estabelecimentos rurais do município de Palmital (SP), cujo universo é composto por 713 estabelecimentos rurais, sendo 529 com prática da agricultura familiar e 184 com prática da agricultura patronal (IBGE, 2006).

As informações foram coletadas por meio da aplicação de formulário ${ }^{4}$, o qual foi respondido por uma amostra estratificada de produtores rurais, proporcional à condição do estabelecimento (familiar ou patronal). Assim, admitindo-se uma população finita e a variável qualitativa 'condição do estabelecimento' como a mais importante para o estudo, o tamanho amostral foi definido em 85 estabelecimentos rurais, sendo 63 com prática da agricultura familiar e 22 com prática da agricultura patronal, a partir de uma margem de erro de $10 \%$ e um nível de confiança de $95,5 \%$ (MARTINS; DOMINGUES, 2011).

Uma lista de estabelecimentos rurais do município de Palmital (SP) foi fornecida pela Casa da Agricultura do referido município e, a partir desta lista, foram selecionados estabelecimentos rurais familiares e patronais de forma aleatória simples ${ }^{5}$, até a composição da amostra planejada, resguardando-se o nome dos produtores responsáveis pelos estabelecimentos ou quaisquer dados que os identificassem quando da tabulação e análise dos dados. O formulário da pesquisa foi elaborado com variáveis explicativas (qualitativas e quantitativas) relativas ao perfil dos estabelecimentos rurais do município de Palmital (SP), conforme Quadro 1 a seguir.

4 Formulário é um instrumento de coleta de dados, cujo sistema consiste em obter informações de modo direto com o entrevistado, por meio de um roteiro de perguntas preenchido pelo entrevistador, no momento da entrevista.

5 A amostragem casual ou aleatória simples pode ser realizada numerando-se a população de 1 a $n$ e sorteando-se $k$ números dessa sequência (usando um dispositivo aleatório qualquer) que corresponderão aos elementos pertencentes à amostra (MONTGOMERY et al., 2012). 
Quadro 1. Código e descrição das variáveis explicativas pesquisadas

(Continua)

\begin{tabular}{|l|l|}
\hline Código & Descrição da Variável \\
\hline ARTER & Área total do estabelecimento rural em hectares (ha). \\
\hline ACAT & $\begin{array}{l}\text { Proporção entre a área destinada a culturas e área total do estabelecimento } \\
\text { rural. }\end{array}$ \\
\hline ACPAT & $\begin{array}{l}\text { Proporção entre a área destinada a culturas permanentes e área total do } \\
\text { estabelecimento rural. }\end{array}$ \\
\hline ACTAT & $\begin{array}{l}\text { Proporção entre a área destinada a culturas temporárias e área total do } \\
\text { estabelecimento rural. }\end{array}$ \\
\hline APAT & $\begin{array}{l}\text { Proporção entre a área destinada a pastagens e área total do estabeleci- } \\
\text { mento rural. }\end{array}$ \\
\hline AMFNAT & $\begin{array}{l}\text { Proporção entre a área de matas e florestas naturais e área total do estabe- } \\
\text { lecimento rural. }\end{array}$ \\
\hline AMFPAT & $\begin{array}{l}\text { Proporção entre a área de matas e florestas plantadas e área total do esta- } \\
\text { belecimento rural. }\end{array}$ \\
\hline APRAT & Proporção entre área própria e área total dos estabelecimentos rurais. \\
\hline ATRAT & $\begin{array}{l}\text { Proporção entre área tomada em arrendamento e área total dos estabele- } \\
\text { cimentos rurais. }\end{array}$ \\
\hline PROVEAN & Produção vegetal e animal. \\
\hline REPROVE & Renda da produção vegetal. \\
\hline REPROVEHA & Renda da produção vegetal por hectare. \\
\hline REPROVEPER & Renda da produção vegetal permanente. \\
\hline REPROVPHA & Renda da produção vegetal permanente por hectare. \\
\hline REPROVETEM & Renda da produção vegetal temporária. \\
\hline REPROVTHA & Renda da produção vegetal temporária por hectare. \\
\hline REPROAN & Renda da produção animal. \\
\hline REPROANHA & Renda da produção animal por hectare. \\
\hline REVTO & Proporção entre a renda vegetal e a renda total. \\
\hline REANTO & Proporção entre a renda animal e a renda total. \\
\hline MOAT & Proporção entre a mão de obra e a área total do estabelecimento rural. \\
\hline TCPROD & Total dos custos de produção. \\
\hline CPROHAP & Custos de produção por hectare produzido. $2 *$ \\
\hline SP1 & SP2. \\
\hline SP2 & Sistema \\
\hline
\end{tabular}


(Conclusão)

\begin{tabular}{|c|c|}
\hline Código & Descrição da Variável \\
\hline SP3 & Sistema de Produção $3^{*}$. \\
\hline SP4 & Sistema de Produção $4^{*}$. \\
\hline SP5 & Sistema de Produção 5*. \\
\hline SP6 & Sistema de Produção 6*. \\
\hline SP7 & Sistema de Produção $7^{*}$. \\
\hline SP8 & Sistema de Produção 8*. \\
\hline SP9 & Sistema de Produção 9*. \\
\hline SP10 & Sistema de Produção $10^{*}$. \\
\hline SP11 & Sistema de Produção 11*. \\
\hline SP12 & Sistema de Produção $12 *$. \\
\hline SP13 & Sistema de Produção $13^{*}$. \\
\hline SP14 & Sistema de Produção $14^{*}$. \\
\hline SP15 & Sistema de Produção $15^{*}$. \\
\hline SP16 & Sistema de Produção 16*. \\
\hline SP17 & Sistema de Produção 17*. \\
\hline SP18 & Sistema de Produção 18*. \\
\hline SP19 & Sistema de Produção 19*. \\
\hline SP20 & Sistema de Produção $20 *$. \\
\hline SP21 & Sistema de Produção $21^{*}$. \\
\hline SP22 & Sistema de Produção $22 *$. \\
\hline SP23 & Sistema de Produção $23^{*}$. \\
\hline SP24 & Sistema de Produção $24^{*}$. \\
\hline SP25 & Sistema de Produção $25^{*}$. \\
\hline SP26 & Sistema de Produção 26*. \\
\hline
\end{tabular}

* Variável dummy onde foi atribuído código um aos produtores que possuem o sistema de produção e código zero aos produtores que não o possuem.

Fonte: Elaborada pelos autores a partir de dados de pesquisa. 


\subsection{ANÁLISE ESTATÍSTICA - MODELO DE REGRESSÃO LINEAR MÚLTIPLO}

Suponha que uma variável dependente (reposta) $Y$ esteja relacionada com um conjunto de $k$ variáveis independentes (explicativas ou preditoras) $\mathbf{X}=\left(X_{1}, X_{2}, \ldots, X_{k}\right)$, que podem ser numéricas ou não, e que uma função relacione tais variáveis, $Y=F(\mathbf{X})=\beta_{0}+\beta_{1} x_{1}+\beta_{2} x_{2}+\ldots \ldots .+\beta_{k} x_{k}$. Desta forma, tem-se um modelo de regressão linear múltiplo dado por:

$$
Y=E(Y \mid \mathbf{X}=\mathbf{x})+\varepsilon
$$

onde $E(Y \mid \mathbf{X}=\mathbf{x})=\beta_{0}+\beta_{1} x_{1}+\beta_{2} x_{2}+\ldots \ldots+\beta_{k} x_{k}$ e $\varepsilon$ é uma variável aleatória (erro aleatório) com média zero e variância $s^{2}$. Os erros, por hipótese, são não correlacionados e, para fins de inferência, é feita a suposição adicional de normalidade dos mesmos.

Neste trabalho, os valores dos coeficientes $\beta_{0}, \beta_{1}, \beta_{2}, \ldots, \beta_{k}$ da equação de regressão (1) são estimados pelo método dos mínimos quadrados ordinários, que busca encontrar os coeficientes (ou estimativas) $b_{0}, b_{1}, b_{2}, \ldots, b_{k}$ que minimizem a soma dos quadrados dos erros (diferenças entre os valores observados de $Y$, e os obtidos a partir do modelo ajustado, $\hat{Y}$ ). Assim, os estimadores de mínimos quadrados do vetor de coeficientes de regressão $\mathbf{B}=\left[\beta_{0}, \beta_{1}, \ldots, \beta_{k}\right]$ são dados por $\hat{\mathbf{B}}=\left(\mathbf{X}^{\prime} \mathbf{X}\right)^{-1} \mathbf{X}^{\prime} \mathbf{Y}=\left[b_{0}, b_{1}, b_{2}, \ldots, b_{k}\right]^{\prime}$, e o vetor dos valores ajustados é representado por $\hat{\mathbf{Y}}=\mathbf{X} \hat{\mathbf{B}}=\mathbf{X}\left(\mathbf{X}^{\prime} \mathbf{X}\right)^{-1} \mathbf{X}^{\prime} \mathbf{Y}$ onde $\mathbf{Y}$ é o vetor de observações de tamanho $n$ e $\mathbf{X}$ é a matriz dos preditores (MONTGOMERY; PECK; VINING, 2012).

Testa-se, então, a significância do modelo por meio do teste de hipótese para a existência de regressão linear múltipla. Sendo $\alpha$ o nível de significância (ou limite do erro) do teste, então, conclui-se que existe regressão linear múltipla, ou seja, que o modelo pode explicar e prever a variável $Y$ se $p$-valor (probabilidade de significância obtida por meio das informações amostrais) for menor ou igual a $\alpha$ . De forma similar, o processo de seleção de variáveis preditoras para o modelo de regressão ajustado é realizado por meio de testes de hipóteses da existência dos parâmetros $\beta_{0}, \beta_{1}, \beta_{2}, \ldots, \beta_{k}$. Sendo $\alpha$ o nível de significância (ou limite do erro) de cada teste, então, conclui-se que $\beta_{i}$ é diferente de zero se $p$-valor (probabilida- 
de de significância obtida por meio das informações amostrais) for menor ou igual a $\alpha$ (MARTINS; DOMINGUES, 2011).

Uma vez definido o modelo e obtidas as estimativas de seus parâmetros, avalia-se a qualidade do ajuste deste aos dados por meio do coeficiente de determinação múltipla, que mede a porcentagem da variação de $Y$ que é explicada pelo modelo. Este coeficiente é dado por $R^{2}=\sum_{i=1}^{n}\left(\hat{Y}_{i}-\bar{Y}\right)^{2} / \sum_{i=1}^{n}\left(Y_{i}-\bar{Y}\right)^{2}$, onde $0 \leq R^{2} \leq 1$ ou $0 \% \leq R^{2} \leq 100 \%$. Quanto mais próximo de um ou de $100 \% R^{2}$ estiver, melhor será a adequabilidade do modelo de regressão linear múltiplo aos dados (MARTINS; DOMINGUES, 2011).

Existem três medidas auxiliares que podem ser utilizadas na verificação dos pressupostos (ou restrições) da regressão: diagnóstico de multicolinearidade ${ }^{6}$, análise da ausência de autocorrelação serial dos resíduos (diferença entre os valores reais e os valores preditos) e da existência de normalidade destes. Para o diagnóstico de multicolinearidade utiliza-se a estatística Variance Inflation Factor (VIF), a qual é calculada estimando-se cada variável independente como se esta fosse dependente, regredindo-a em relação às demais e obtendo-se o valor $\left(1-R^{2}\right)$ de tal regressão. Se $\mathrm{VIF}<1$, não existe multicolinearidade; $\mathbf{1} \leq \mathrm{VIF} \leq \mathbf{1 0}$, a multicolinearidade é aceitável; VIF $>10$, a multicolinearidade é problemática. A análise da autocorrelação serial se faz por meio do teste de Durbin-Watson (D-W). Sendo $\alpha$ o nível de significância (ou limite do erro) do teste, então, conclui-se que existe ausência de autocorrelação serial dos resíduos se a estatística D-W estiver próxima a 2 . E verifica-se ainda a normalidade dos resíduos graficamente e por meio do teste de Kolmogorov-Smirnov (K-S). Sendo $\alpha$ o nível de significância (ou limite do erro) do teste, então, conclui-se que a distribuição dos resíduos é normal se a estatística K-S, calculada a partir da distribuição $D$ (distância euclidiana máxima), for menor do que o valor crítico (tabelado), ou equivalentemente, se o p-valor (probabilidade de significância obtida por meio das informações amostrais) for maior do que $\alpha$ (MONTGOMERY; PECK; VINING, 2012).

6 A existência da correlação cruzada entre variáveis independentes é chamada de multicolinearidade. Quando existe multicolinearidade, o processo de estimação é insatisfatório e os coeficientes sobre cada uma das variáveis independentes tornam-se difíceis de serem interpretados isoladamente, pois as variáveis colineares não proporcionam informação suficiente para estimar seus efeitos separados (MONTGOMERY; PECK; VINING, 2012). 


\section{RESULTADOS E DISCUSSÕES}

\subsection{ANÁLISE ESTATÍSTICA DA RENDA AGRÍCOLA DOS AGRICULTORES FAMILIARES}

Primeiramente foi feita uma análise de regressão considerando a variável resposta 'renda agrícola dos produtores familiares' ( $Y=$ RENTO) e as variáveis explicativas (ou independentes) do Quadro 1. Para assegurar a inexistência de multicolinearidade entre tais variáveis, calculou-se a estatística VIF e, retirando da análise as variáveis que apresentaram VIF $>10$, renda da produção vegetal (REPROVE), renda da produção vegetal permanente por bectare (REPROVEPHA), renda da produção vegetal temporária (REPROVETEM), renda da produção animal (REPROAN) e custos de produção por bectare produzido (CPROHAP), ficou afastada a existência de multicolinearidade problemática e confirmada a capacidade preditiva de um modelo de regressão linear por meio das demais variáveis.

Desta forma, com a finalidade de explicar o quanto da variação da renda agrícola (RENTO) dos agricultores familiares se deve ao conjunto de variáveis independentes considerado, foi ajustado um modelo de regressão linear múltiplo a partir dos dados amostrais. Para um nível de significância de 10\%, o modelo de regressão final ajustado aos dados, conforme Tabela 1 , foi: RENTO $=119919+$ 2401 ARTER + 13,97 REPROVEHA + 0,2774 REPROVEPER + 16,71 REPROVTHA + 4,33 REPROANHA - 236451 REVTO + 0,2466 TCPROD + 67341 SP1 + 22138 SP2 +69964 SP $4+26642$ SP6 + 25183 SP8 + 75667 SP12 + 56173 SP18.

Tabela 1. Resumo da análise de regressão linear múltipla (agricultura familiar)

(Continua)

\begin{tabular}{lcccc}
\hline Preditor & Coef & T & p-valor & VIF \\
\hline Constante & 119919 & 3,64 & 0,001 & - \\
ARTER & 2401 & 9,85 & 0,000 & 2,74 \\
REPROVEHA & 13,97 & 5,66 & 0,000 & 4,66 \\
REPROVEPER & 0,2774 & 3,32 & 0,002 & 3,22 \\
REPROVTHA & 16,71 & 5,28 & 0,000 & 2,11 \\
REPROANHA & 4,33 & 3,30 & 0,002 & 3,76 \\
REVTO & 236451 & $-6,81$ & 0,000 & 1,96
\end{tabular}


(Conclusão)

\begin{tabular}{|c|c|c|c|c|c|}
\hline Preditor & Coef & $\mathrm{T}$ & p-valor & VIF & \\
\hline TCPROD & 0,2466 & 3,24 & 0,002 & 4,14 & \\
\hline SP1 & 67341 & 1,97 & 0,054 & 1,12 & \\
\hline SP2 & 22138 & 1,88 & 0,065 & 1,52 & \\
\hline SP4 & 69964 & 1,96 & 0,055 & 1,21 & \\
\hline SP6 & 26642 & 2,58 & 0,012 & 1,69 & \\
\hline SP8 & 25183 & 1,93 & 0,059 & 1,76 & \\
\hline SP12 & 75667 & 2,19 & 0,032 & 1,13 & \\
\hline SP18 & 56173 & 1,65 & 0,100 & 1,10 & \\
\hline$S=32204,9$ & & -quad $=95,59 \%$ & R-quad (ajust) $=$ & $4,52 \%$ & \\
\hline \multicolumn{6}{|c|}{ Análise de Variância (ANOVA) } \\
\hline Variação & GL & SQ & QM & $\mathrm{F}$ & $\mathrm{p}$-valor \\
\hline Regressão & 14 & 1303272288897,22 & 93090877778,37 & 89,78 & 0,001 \\
\hline Erro Residual & 58 & 60137710064,26 & 1036857070,07 & & \\
\hline Total & 72 & 1363409998961,48 & & & \\
\hline
\end{tabular}

Estatística de Durbin-Watson $=1,95$

Quanto à significância do modelo de regressão, como p-valor $<10 \%$ (vide estatística $\mathrm{F}$ e respectivo p-valor na ANOVA da Tabela 1), há evidências de que o modelo pode explicar e prever a variável resposta (RENTO). Da mesma forma,

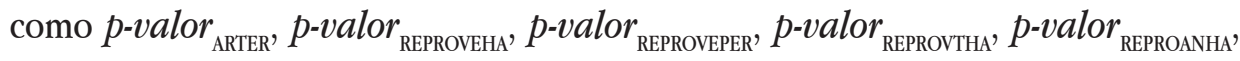

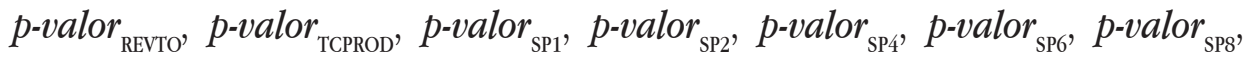
$p$-valor ${ }_{\text {PP12 }}$ e p-valor $r_{\text {SP18 }}$ são menores que $10 \%$ (vide estatísticas T e respectivos p-valores da Tabela 1), os parâmetros de tais variáveis confirmaram-se como significativos para o modelo.

De acordo com a ordem crescente dos valores (em módulo) das estatísticas dos testes, obteve-se que as variáveis independentes área total do estabelecimento rural em hectares (ARTER), proporção entre a renda vegetal e a renda total (REVTO), renda da produção vegetal por hectare (REPROVEHA), renda da produção vegetal temporária por hectare (REPROVTHA), renda da produção vegetal permanente (REPROVEPER), renda da produção animal por hectare (REPROANHA), total dos 
custos de produção (TCPROD), sistema de produção 6: soja e milho safrinba (SP6), sistema de produção 12: cana-de-açúcar, suínos e galinhas (SP12), sistema de produção 1: banana nanica (SP1), sistema de produção 4: banana nanica e peixe Tilápia (SP4), sistema de produção 8: soja, milbo safrinba e cana-de-açúcar (SP8), sistema de produção 2: cana-de-açúcar (SP2) e sistema de produção 18: soja, milbo safrinba, suinos e galinhas (SP18), foram as que mais influenciaram a renda agrícola dos produtores familiares, respectivamente.

O grau de ajustamento do modelo foi avaliado baseado no coeficiente de determinação múltipla (R-quad na Tabela 1), cujo valor estabelece que 95,59\% da variação da renda dos estabelecimentos rurais (com agricultura familiar) se explicam pelas variáveis independentes significativas. Além disso, o teste de KolmogorovSmirnov ( $p$-valor $>0,10$ ) para os resíduos padronizados confirmou a normalidade destes, conforme Gráfico 1. Finalmente, por meio da estatística de DurbinWatson, que é aproximadamente igual a 2, observou-se que os resíduos não estão autocorrelacionados.

A variável REVTO apresentou coeficiente negativo, o que significa que esta se movimenta em sentido contrário ao da RENTO, quando mantidas as demais variáveis constantes. Por outro lado, as demais variáveis, ARTER, REPROVEHA, REPROVTHA, REPROANHA, TCPROD, SP1, SP2, SP4, SP6, SP8, SP12 e SP18 apresentaram coeficientes positivos, indicando que estas se movimentam (individualmente) no mesmo sentido da RENTO, quando mantidas as demais variáveis constantes. 


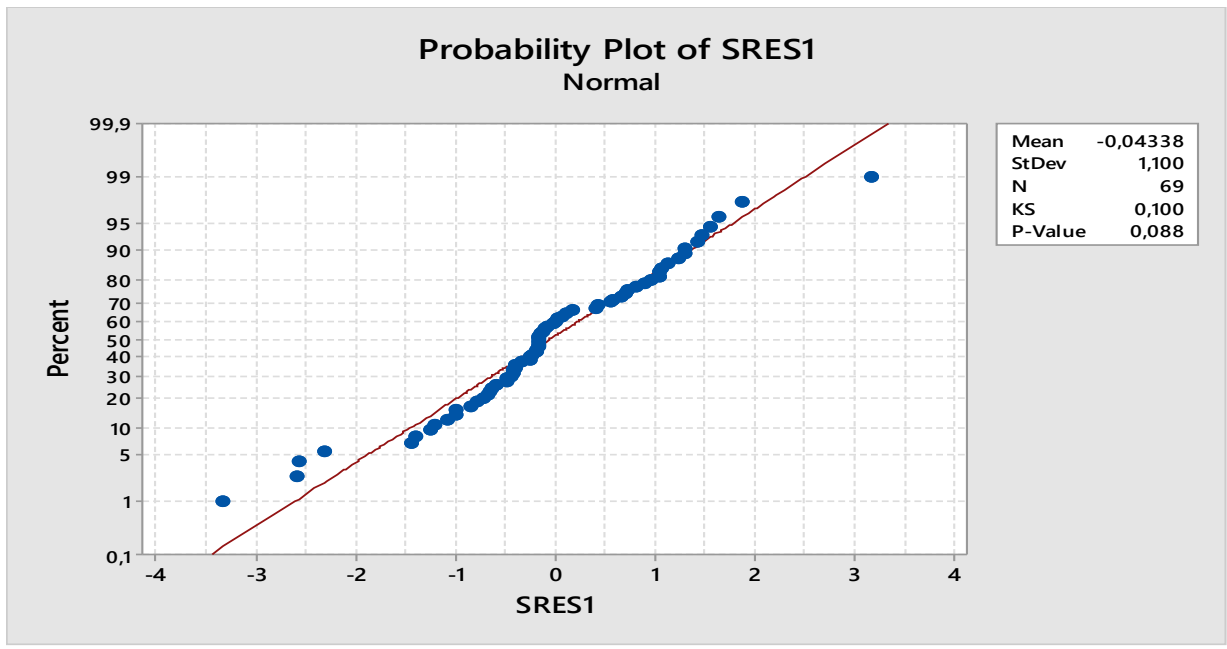

Gráfico 1. Teste de normalidade dos resíduos padronizados (agricultura familiar) Fonte: Do autor.

\subsection{ANÁLISE ESTATÍSTICA DA RENDA AGRÍCOLA DOS AGRICULTORES PATRONAIS}

De maneira semelhante à análise feita com os produtores familiares, fezse uma análise com os produtores patronais, onde inicialmente, para assegurar a inexistência de multicolinearidade entre as variáveis independentes, foi calculada a estatística VIF considerando todas as variáveis independentes do Quadro 1. Retirando da análise as variáveis proporção entre áreas destinadas a culturas permanentes $e$ área total do estabelecimento rural (ACPAT), proporção entre áreas destinadas a culturas temporárias e área total do estabelecimento rural (ACTAT), proporção entre área de matas e florestas plantadas e área total do estabelecimento rural (AMFPAT), renda da produção vegetal permanente (REPROVEPER), renda da produção vegetal permanente por hectare (REPROVEPEHA) e total dos custos de produção (TCPROD), (que apresentaram VIF > 10), ficou afastada a existência de multicolinearidade inicial e confirmada a capacidade preditiva de um modelo de regressão linear por meio das demais variáveis.

Assim, com a finalidade de explicar o quanto da variação na renda agrícola (RENTO) dos agricultores patronais se deve ao conjunto de variáveis independentes ou explicativas considerado, foi ajustado um modelo de regressão linear múltiplo 
a partir dos dados amostrais. Considerando um nível de significância de 10\%, o modelo de regressão final ajustado aos dados, conforme Tabela 2, foi: RENTO = $-394583+2898$ ARTER + 182,0 REPROVEHA + 0,2582 REPROVETEM - 134,3 CPROHAP +506533 SP9.

Tabela 2. Resumo dos resultados da análise de regressão linear múltipla (agricultura patronal)

\begin{tabular}{llllll}
\hline Preditor & Coef & T & p-valor & VIF & \\
\hline Constante & -394583 & $-3,52$ & 0,002 & & \\
ARTER & 2898 & 15,01 & 0,000 & 5,44 & \\
REPROVEHA & 182 & 7,97 & 0,000 & 1,84 & \\
REPROVETEM & 0,2582 & 4,88 & 0,000 & 6,36 & \\
CPROHAP & $-134,3$ & $-2,93$ & 0,009 & 1,56 & \\
SP9 & 506533 & 4,78 & 0,000 & 1,51 & \\
\multicolumn{7}{l}{} & & & & \\
\hline S $=84600,8$ & R-quad $=99,23 \%$ & R-quad (ajust) $=99,03 \%$ & \\
\hline Análise de Variância (ANOVA) & & & & \\
Variação & GL & SQ & QM & F & p-valor \\
Regressão & 5 & 17580711528661,3 & 3516142305732,2 & 488,06 & 0,002 \\
Erro Residual & 19 & 136880600436,9 & 7204242128,2 & & \\
Total & 24 & 17717592129098,2 & & & \\
\hline
\end{tabular}

Estatística de Durbin-Watson $=1,44$

Quanto à significância do modelo de regressão, como p-valor $<10 \%$ (vide estatística $\mathrm{F}$ e respectivo p-valor na ANOVA da Tabela 2), há evidências de que o modelo pode explicar e prever a variável resposta (RENTO). Da mesma forma, como $p$-valor $r_{\text {ARTER }}$, $p$-valor $r_{\text {REPROVEHA }}$ p-valor $r_{\text {REPROVETEM }}, p$-valor $r_{\text {CPROHAP }}$, e $p$-valor $r_{\text {SP9 }}$ são menores que $10 \%$ (vide estatísticas T e respectivos p-valores da Tabela 2), os parâmetros de tais variáveis confirmaram-se como significativos para o modelo.

Logo, assegurou-se que, de acordo com a ordem crescente dos valores (em módulo) das estatísticas dos testes, as variáveis independentes, área total do estabelecimento rural em bectares (ARTER), renda da produção vegetal por bectare (REPROVEHA), renda da produção vegetal temporária (REPROVETEM), sistema de produção 9: soja, milho safrinha e mandioca (SP9) e custos de produção por 
bectare produzido (CPROHAP), foram as que mais contribuíram para a formação da renda agrícola de produtores patronais, respectivamente.

O grau de ajustamento do modelo foi avaliado baseado no coeficiente de determinação múltipla (R-quad na Tabela 3), cujo valor estabelece que 99,23\% da variação da RENTO dos estabelecimentos rurais (com agricultura patronal) se explicam pelas variáveis independentes significativas. Além disso, o teste de Kolmogorov-Smirnov ( $p$-valor $>0,15$ ) para os resíduos padronizados confirmou a normalidade destes, conforme Gráfico 2. Finalmente, por meio da estatística de Durbin-Watson, que é aproximadamente igual a 2, observou-se que os resíduos não estão autocorrelacionados.

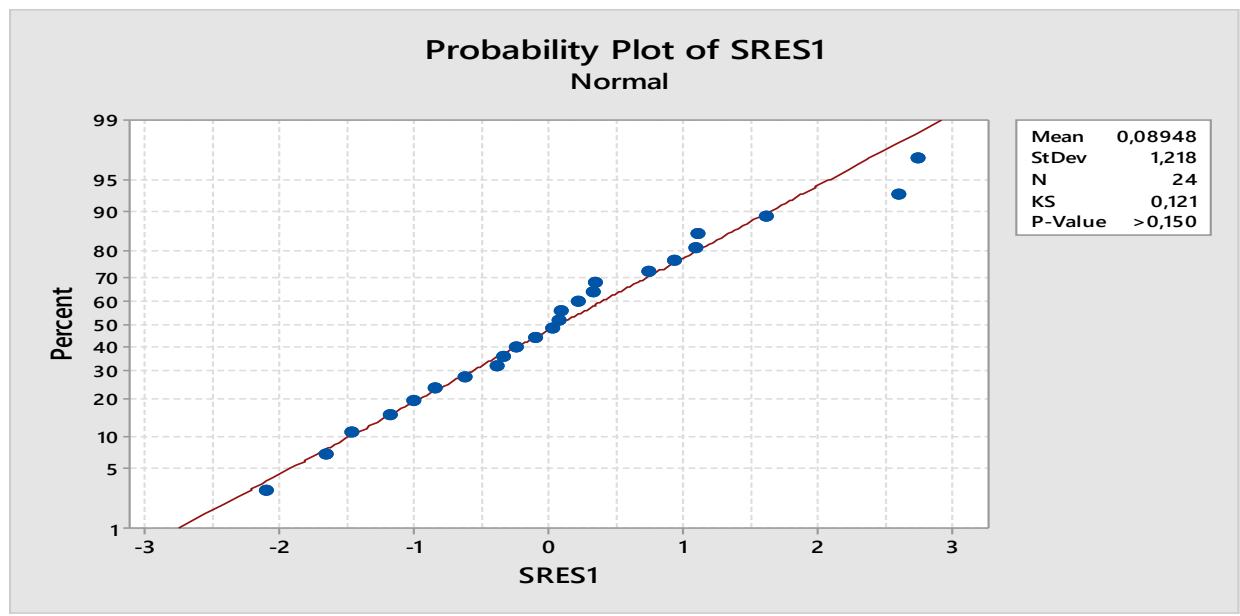

Gráfico 2. Teste de normalidade dos resíduos padronizados (agricultura patronal) Fonte: Elaborado pelos autores a partir dos dados de pesquisa.

De acordo com a análise exposta no Gráfico 2, a variável CPROHAP apresentou coeficiente negativo, o que significa que esta se movimenta (individualmente) em sentido oposto ao da RENTO, quando mantida as demais variáveis constantes. Por outro lado, as variáveis ARTER, REPROVEHA, REPROVETEM e SP9 apresentaram coeficientes positivos, o que indica que estas se movimentam no mesmo sentido da RENTO, quando mantidas as demais variáveis constantes. 


\subsection{DISCUSSÕES}

De acordo com a análise estatística e os resultados obtidos, observa-se que há indícios de que a diversidade dos sistemas de produção melhora a renda dos agricultores familiares do município de Palmital (SP). Diversidade que Schneider (2006) apresenta como estratégia de produção e das trajetórias históricas que influenciam a manutenção destes agricultores nas mais diversas regiões do Brasil.

Analisando a diversidade dos sistemas de produção que influenciam a renda dos produtores familiares, verifica-se a interação entre os sistemas e subsistemas apresentada por Von Bertalanffy (2013). Essa diversidade mostra que a renda é um fator de manutenção e reprodução destes produtores no município. Além disso, o aumento de um desses sistemas de produção, ou seja, a atribuição do mesmo a mais áreas, poderia contribuir para melhorar a renda dos agricultores familiares.

Outra variável significativa à agricultura familiar foi a área total do estabelecimento ruralem bectares (ARTER), sendo esta a variável que mais influenciou a renda agrícola de tais produtores. Por outro lado, ela também foi significativa à agricultura patronal, uma vez que quanto maior a área do estabelecimento, maior será a renda esperada.

Em seguida, as variáveis renda da produção vegetal por bectare (REPROVEHA) e renda da produção vegetal temporária por hectare (REPROVTHA), respectivamente, influenciaram a renda agrícola dos produtores familiares, pois a agricultura prevalece sobre a pecuária no município, e as culturas temporárias prevalecem sobre as culturas permanentes no caso dos agricultores familiares. Mesmo com essa predominância, a variável renda das culturas permanentes (REPROVEPER) também influencia a renda agrícola, pois dependendo do produto (por exemplo a banana nanica), podem ocorrer grande produção e preço favorável de comercialização em relação a outras culturas temporárias, como a cana-de-açúcar e a mandioca.

Outra variável que se destacou foi total dos custos de produção (TCPROD) que, quanto maior, mais afeta (aumenta) a renda dos agricultores familiares do município, que possuem alto custo de produção, pois não compram em grandes escalas, perdendo assim o poder de negociação. 
Dentre a diversidade de sistemas de produção encontrados no município, sete se destacaram como as variáveis que mais afetaram a renda dos agricultores familiares, todos de forma positiva. Houve um destaque para os sistemas de produção que incluíam as culturas da soja e do milho safrinha, além da presença da produção animal, o que não se observou para os agricultores patronais.

Já em relação aos produtores patronais do município de Palmital (SP), além da representatividade da variável área total do estabelecimento rural em bectares (ARTER), destacou-se ainda a variável renda da produção vegetal por bectare (REPROVEHA) indicando que, além do tamanho da área, o produtor patronal deve se preocupar com a produção por hectare, ou seja, o suporte técnico e o uso de novas tecnologias de produção são importantes para se obter alta produtividade de sacas e/ou toneladas do produto por hectare em seus estabelecimentos rurais.

Já a variável renda da produção vegetal temporária (REPROVETEM) mostra que a renda da produção patronal no município estudado sofre influências de culturas temporárias, o que mostra que a diversidade de produção também influencia positivamente a renda dos produtores patronais do município. Tal fato se comprova pela significância do sistema de produção composto pela soja, milho safrinha e mandioca à renda agrícola de tais produtores. As duas primeiras são fortes culturas no município, no entanto, a mandioca é conhecida como produto da agricultura familiar. Neste caso, a análise estatística aponta que a aplicação deste sistema de produção em mais áreas poderia melhorar a renda dos agricultores patronais. Em relação à variável custo de produção por hectare produzido (CPROHAP) observouse que, quanto maior a renda, menor serão os custos de produção por hectare, já que ao se produzir em grandes escalas, diminuem-se os custos fixos de produção.

Portanto, tais resultados mostram que os produtores patronais do município poderiam obter melhores rendas com o aumento das áreas de produção, aumento da produção por hectare por meio de apoio técnico e de novas tecnologias de produção, o que consequentemente levará a diminuição dos custos de produção por hectare. Revelou-se ainda como fator positivo ao aumento da renda a produção de culturas temporárias, o que levaria à diversificação de sua produção.

Percebe-se que em relação ao trabalho de Silva et al. (2008), houve uma diferença entre as análises. Enquanto esses autores realizaram uma análise para a 
renda bruta dos produtores rurais como função da área das propriedades, índices de rendimento das atividades, renda não agrícola, aposentadorias e índices de diversificação, aqui se fez uma análise da renda agrícola, e dentre as variáveis independentes analisadas em ambos os trabalhos, apenas área dos estabelecimentos rurais é comum aos dois trabalhos. A maioria das variáveis independentes consideradas por Silva et al. (2008) não está presente na análise realizada em Palmital (SP), uma vez que fogem dos objetivos deste trabalho que está relacionado somente à renda agrícola.

Já em comparação ao trabalho realizado por Mochiuti et al. (2011), cujo objeto de pesquisa também foi a renda bruta total dos produtores rurais de Tupã (SP), algumas variáveis independentes foram semelhantes. Em ambos os trabalhos foram levantados o perfil das famílias dos produtores rurais por meio das variáveis (número de integrantes, idade, sexo, ocupação, escolaridade), a estrutura da produção agropecuária (área e tecnologia de produção animal e vegetal), e a formação da renda. No trabalho de Mochiuti et al. (2011), as variáveis existência de assistência técnica, proporção entre a área destinada à cultura e a área total do estabelecimento rural; proporção entre a área arrendada e/ou, em parceria em relação à área do estabelecimento rural; número de animais ligados à bovinocultura; e índice de diversificação foram significativas.

No trabalho realizado por Pinto et al. (2016), que analisou a renda bruta total de estabelecimentos rurais familiares da região Nova Alta Paulista, considerouse proporção entre a renda agrícola e a renda bruta; área total do estabelecimento rural; proporção entre a renda obtida através da aposentadoria e/ou, pensão e a renda bruta; proporção entre o número de animais totais e a área total do estabelecimento rural; e índice de diversificação como significativas à composição da renda bruta total de tais produtores. As variáveis consideradas nos dois trabalhos mencionados também foram analisadas neste trabalho, porém, não afetaram a renda agrícola de forma que pudessem ser explicativas ao modelo de regressão encontrado.

Pôde-se perceber que a principal diferença entre os trabalhos citados e este é que aqui foram abordadas variáveis que afetam a renda agrícola dos produtores rurais do município estudado, tendo a preocupação em não abordar outras rendas que são fundamentais para formação da renda bruta total destes produtores, objetivando 
analisar se estes produtores estão se capitalizando ou não, especificamente com a renda agrícola de seus estabelecimentos rurais. Além disso, o número de variáveis analisadas para se chegar aos resultados finais foi bem maior em relação aos demais trabalhos (quarenta e nove), mesmo o objetivo se restringindo à renda agrícola, tamanha complexidade que envolve o tema.

\section{CONSIDERAÇÕES FINAIS}

A caracterização da estrutura produtiva dos agricultores (familiares e patronais) do município de Palmital (SP) proporcionou resultados importantes, e levaram à identificação de quais fatores influenciam a renda agrícola destes agricultores, e que são bem característicos de cada tipo de agricultor.

De acordo com os modelos estatísticos propostos, descrever a renda agrícola de agricultores patronais envolve aspectos relacionados à área do estabelecimento rural, a rendas de produção vegetal, ao custo de produção e a um sistema de produção específico que é composto por culturas relevantes ao município. A renda agrícola de agricultores familiares também é influenciada pela área do estabelecimento rural e pelo custo de produção, no entanto, envolvem diversos fatores, tais como renda das produções temporárias e permanentes, e a influência de sete sistemas de produção dentre todos encontrados no município, mostrando a importância e relevância da diversidade produtiva na composição da renda de tais produtores.

\section{REFERÊNCIAS}

ABRAMOVAY, R. O futuro das regióes rurais. Porto Alegre: Ed. da UFRGS, 2010.

ALTAFIN, I. Reflexões sobre o conceito de agricultura familiar: conteúdo aplicado durante o $3^{\circ}$ módulo do curso regional de formação político-sindical da região nordeste 2007. Disponível em: http://mstemdados.org/sites/default/files/Reflex\%C3\%B5es\%20sobre\%20o\%20conceito\%20de\%20agricultura\%20familiar\%20-\%20 Iara\%20Altafin\%20-\%202007_0.pdf. Acesso em: 5 set. 2014.

ÁREA de PD está dobrando em São Paulo. Plantio Direto, p. 65-67, jun. 2000.

BUAINAIN, A. M.; ALVES, E.; SILVEIRA, J. M.; ZANDE, R. N. O mundo rural no 
Brasil do século 21: a formação de um novo padrão agrário e Agrícola. Brasília: Embrapa. 2014.

CEPEA. PIB do agronegócio brasileiro. Disponível em: http://www.cepea.esalq. usp.br/br/pib-do-agronegocio-brasileiro.aspx. Acesso em: 2 mar. 2017.

DELGADO, G. C.; BERGAMASCO, S. M. P. P. (org.). Agricultura familiar brasileira: desafios e perspectivas de futuro. Brasília: SEAF, 2017.

DELGADO, N. Agronegócio e agricultura familiar no Brasil: desafios para a transformação democrática do meio rural. Novos Cadernos NAEA, Belém, v. 15, p. 85-129, 2012. Disponível em: http://www.periodicos.ufpa.br/index.php/ncn/article/ viewArticle/868. Acesso em: 8 jun. 2017.

FURLANETO, F. de P. B.; KANTHACK, R. A. D.; BONISSONI, K. C. O agronegócio da mandioca na região paulista do Médio Paranapanema. Análise e Indicadores do Agronegócio, v. 1, n. 4, abr. 2006.

IBGE. Característica da população. 2012. Disponível em: http://www.ibge.gov.br/ ibgeteen/pesquisas/demograficas.html. Acesso em: 5 dez. 2016.

IBGE. Censo Agropecuário 2006. Disponível em: https://ww2.ibge.gov.br/home/ estatistica/economia/agropecuaria/censoagro/2006_segunda_apuracao/default. shtm. Acesso em: 16 dez. 2016.

LEITE, S.; MEDEIROS, L. Agronegócio. In: CALDART, R. et al. (org.). Dicionário da educação do campo. Rio de Janeiro: Escola Politécnica de Saúde Joaquim Venâncio; São Paulo: Expressão Popular, 2012.

LIMA, Arlindo P. de et al. Administração da unidade de produção familiar modalidade de trabalho com agricultores. 3. ed. Ijuí, RN: Unijuí, 2005.

MARTINS, G. A. M.; DOMINGUES, O. Estatística geral e aplicada. 4. ed. São Paulo: Atlas, 2011. $662 \mathrm{p}$.

MOCHIUTI, J. C.; PINTO, L. de B.; OLIVEIRA, S. C.; MAGALHÃES, M. M. Estudo sobre a composição da renda e a lógica de funcionamento dos sistemas de produção 
agropecuários em Tupã-SP. Omnia Exatas, v. 4, n. 2, p. 77-86, 2011.

MONTGOMERY, D. C.; PECK, E. A.; VINING, G. G. Introduction to linear regression analysis. 5. ed. New York: John Wiley \& Sons, 2012. 645 p.

NEVES, D. P. Agricultura familiar: quantos ancoradouros! Disponível em: http:// www2.fct.unesp.br/nera/usorestrito/Agricultura_Familiar.pdf. Acesso em: 13 set. 2014.

NOGAMI, O. Economia. Curitiba: IESDE Brasil, 2012. 246 p.

NUNES, E. M. Reestruturação agrícola, instituições e desenvolvimento rural no nordeste: as dinâmicas regionais e a diversificação da agricultura familiar no Pólo Assu-Mossoró (RN). 2009. Tese (Doutorado em Desenvolvimento Rural) Faculdade de Ciências Econômicas, Universidade Federal do Rio Grande do Sul, Porto Alegre, 2009.

PINTO, L. de B. et al. Um estudo sobre a renda bruta de estabelecimentos rurais familiares de Municípios da Região Nova Alta Paulista, Estado de São Paulo, Brasil. Revista ESPACIOS, v. 37, n. 6, 2016.

SCHNEIDER, S. (org.). A diversidade da agricultura familiar. Porto Alegre: Ed. da Universidade UFRGS, 2006.

SCHNEIDER, S. A pluriatividade na agricultura familiar. 2. ed. Porto Alegre: Ed. da UFRGS, 2009.

SILVA, C. D. et al. Análise da rentabilidade de pequenas propriedades rurais. ANPAD, 2008. Disponível em: http://www.anpad.org.br/admin/pdf/GCT-D1195.pdf. Acesso em: 5 dez. 2016.

UDAETA, M. E. M. et al. Proposição para o interior paulista do planejamento integrado de recursos. São Paulo: GEPEA-USP, 2004.

VON BERTALANFFY, L. Teoria geral dos sistemas: fundamentos, desenvolvimento e aplicações. 7. ed. Petrópolis: Vozes, 2013. 
ZYLBERSZTAJN, D.; NEVES, M. F.; NEVES, E. M. Agronegócio no Brasil. São Paulo: Saraiva, 2005.

Recebido em: 23/02/2018

Aceito em: 29/08/2018 